\title{
Site-Specific Mutagenesis in Escherichia coli by Bulky Exocyclic Amino-Substituted Guanine and Adenine Derivatives in Double-Stranded or Gapped Plasmids
}

\author{
Ki-Young Moon, Ph.D. \\ Department of Clinical Pathology, and Bioindustry and Technology Research Institute, Kwangju Health College, Gwangju, \\ Korea
}

Purpose: 7-Bromomethylbenz[a]anthracene is a $\mathrm{kn}$ own mutagen and carcinogen. The mutagenic potency of its two major DNA adducts, i.e., $N^{2}$-(benz[a]anthracen7-ylmethyl)-2'-deoxyguanosine (b[a]a $\left.{ }^{2} G\right)$ and $N^{6}$-(benz [a]anthracen-7-ylmethyl)-2'-deoxyadenosine (b[a]a $\left.{ }^{6} A\right)$, as well as the simpler benzylated analogs, $N^{2}$-benzyl- $2^{\prime}$ deoxyguanosine $\left(\mathrm{bn}^{2} \mathrm{G}\right)$ and $N^{6}$-benzyl-2'-deoxyadenosine $\left(b n^{6} A\right)$, were determined in $E$. coli.

Materials and Methods: Double-stranded and gapped plasmid vectors were used to determine the mutagenicity of $b[a] a^{2} G, b[a] a^{6} A, b n^{2} G$ and $b n^{6} A$ in $E$. coli. The four, suitably protected, bulky exocyclic amino-substituted adducts were incorporated into 16-base oligodeoxyribonucleotides, in place of normal guanine or adenine residues, which form part of the ATG initiation codon for the lacZ' a-complementation gene. The site-specifically modified oligodeoxyribonucleotides were then incorporated into double-stranded plasmids, which contained uracil residues in the complementary strand in the vicinity of the initiation codon. The uracil residues lead to the creation of a gap in the complementary strand due to the actions of $E$. coli uracil-DNA glycosylase and

\section{INTRODUCTION}

Exposure to low levels of chemical carcinogens in the environment is essentially unavoidable, and their damage to cellular DNA has been recognized in the initiation of carcinogenic processes through mutagenic mechanisms. It is difficult to evaluate how any one carcinogen adduct can alter the structure, or coding properties, of the damaged DNA, as carcinogens can react with a variety of sites on DNA bases, and with several bases simultaneously. Therefore, the synthetic

Correspondence: Ki-Young Moon, Department of Clinical Pathology, Kwangju Health College, 683-3, Shinchang-dong, Gwangsan-gu, Gwangju 506-701, Korea. (Tel) 062-958-7621, 7577, (Fax) 062953-6085, (E-mail) kmoon@www.kjhc.ac.kr

Received December 4, 2002, Accepted February 14, 2003
AP endonuclease. Following the transfection of these plasmid vectors into $E$. coli strain GP102, a lacZ alpha complementing version of the parent strain $A B 1157$, their propensity to induce mutation was investigated.

Results: The percentages of mutant colonies produced by the four modified nucleosides, in both the doublestranded and gapped plasmid vectors, were not significantly different from those produced by the unmodified plasmids. The mutagenicities of the $b[a] a^{2} G$ and $b[a] a^{6} A$ were extremely low, and a totally unexpected result, whereas, those of the $b n^{2} G$ and $b n^{6} A$ were undetectable.

Conclusion: In this $E$. coli site-specific mutagenesis system, these bulky aralkylated adducts exhibited no significant mutagenicities, either with or without SOS induction. (Cancer Research and Treatment 2003;35:75. 80)

Key Words: Mutagenesis, 7-Bromomethylbenz[a]anthracene, Gapped plasmid, Bulky exocyclic amino-substituted adducts, sOS induction, $E$. coli methods for the preparation of oligodeoxyribonucleotides, containing a single specific carcinogen-modified base at defined sites, have been developed, and play key roles in the investigations of individual carcinogen-DNA adducts, and how they induce mutagenic potency in biological systems $(1,2)$.

The environmental pollutant 7-bromomethylbenz[a]anthracene $(7-\mathrm{BrMeBA})$ is a known mutagen and carcinogen $(3 \sim 7)$. The exocyclic $N^{2}$ and $N^{6}$ amino groups of deoxyguanosine and deoxyadenosine in DNA are major sites of aralkylation. $N^{2}$-(benz[a]anthracen-7-ylmethyl)-2'-deoxyguanosine $\quad\left(\mathrm{b}[\mathrm{a}] \mathrm{a}^{2} \mathrm{G}\right)$ and $\mathrm{N}^{6}$-(benz[a]anthracen-7-ylmethyl)-2'-deoxyadenosine (b $[a] a^{6} \mathrm{~A}$ ) are the major modified deoxyribonucleosides produced in DNA by this carcinogen.

This study was based on synthesized oligodeoxyribonucleotides, containing a single carcinogen-modified base at a specific location. To determine the mutagenic potencies of these bulky adducts, and that of the simpler benzylated analogs, i.e., $N^{2}$ benzyl-2'-deoxyguanosine ( $\mathrm{bn}^{2} \mathrm{G}$ ) and $N^{6}$-benzyl-2'-deoxyade- 


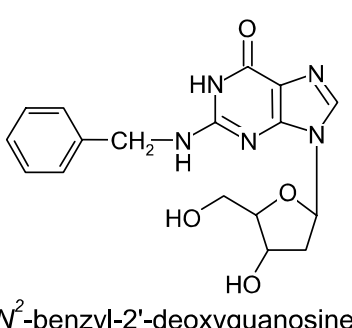

$\left(b n^{2} G\right)$

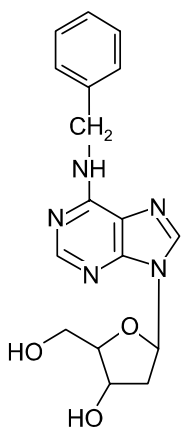

$N^{6}$-benzyl-2'-deoxyadenosine $\left(b n^{6} \mathrm{~A}\right)$

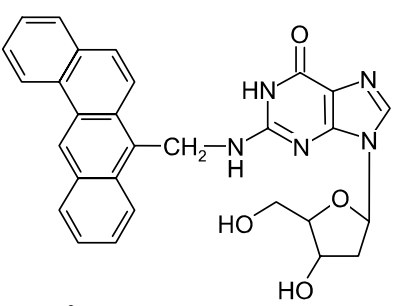

$N^{2}$-(benz[a]anthracen-7ylmethyl)-2'-deoxyguanosine $\left(\mathrm{b}[\mathrm{a}] \mathrm{a}^{2} \mathrm{G}\right)$<smiles>Cc1c2ccccc2cc2c1ccc1ccccc12</smiles><smiles>N[AlH2]</smiles><smiles>Nc1ncnc2[nH]cnc12</smiles><smiles>OCC1OC2CC1C2O</smiles>

$N^{6}$-(benz[a]anthracen-7ylmethyl)-2'-deoxyadenosine $\left(\mathrm{b}[\mathrm{a}] \mathrm{a}^{6} \mathrm{~A}\right)$

Fig. 1. The chemical structures of the four bulky aralkylated nucleosides.

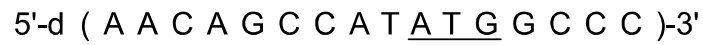

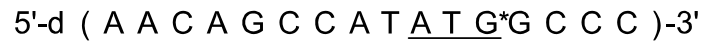

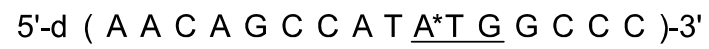

The asterisks mark the positions of the incorporation of the modified guanine and adenine derivatives.

Fig. 2. Synthetic DNA sequences containing N2-aralkylated guanine and N6-aralkylated adenine nucleosides.

nosine $\left(\mathrm{bn}^{6} \mathrm{~A}\right)$, the primary adducts produced by benzyl bromide $(8,9)$, we recently reported on the preparation of $\mathrm{bn}^{2} \mathrm{G}, \mathrm{bn}^{6} \mathrm{~A}$, $\mathrm{b}[\mathrm{a}] \mathrm{a}^{2} \mathrm{G}$ and $\mathrm{b}[\mathrm{a}] \mathrm{a}^{6} \mathrm{~A}$ by the direct aralkylation of $2^{\prime}$-deoxyguanosine and 2'-deoxyadenosine (10) (Fig. 1). These bulky exocyclic amino-substituted adducts were incorporated into 16-base oligodeoxyribonucleotides, in place of normal guanine or adenine residues, which form part of the ATG initiation codon for the lacZ' a-complementation gene, using an in situ activation approach, with automated phosphite triester synthetic methods (10) (Fig. 2). These modified DNA segments were used to determine how specific examples of carcinogen-DNA damage can induce mutations. Following the insertion of modified bases, containing an oligodeoxyribonucleotide, into plasmids, their propensity to induce a mutation can be studied in E. coli.

The mutagenicities of the major DNA adducts produced by 7-BrMeBA, when the modified base was incorporated into plasmid vectors, was examined, and compared, in E. coli. The mutagenicities of these adducts were also compared to those of the simpler benzylated derivatives of guanine and adenine, i.e., $b n^{2} \mathrm{G}$ and $b n^{6} \mathrm{~A}$, in order to observe the effects of increasing the size of the exocyclic amino group substituents on mutagenic potency. This was performed to allow us to broaden our understanding on how substituted structure can influence the mutagenicity of bulky exocyclic amino-substituted adducts. Even though 7-BrMeBA is a known carcinogen, this is the first evaluation of the mutagenicity induced by the site-specific incorporation of its major adducts in E.coli.

This study now reports the site-specific mutagenesis induced by four aralkylated nucleosides in E. coli.

\section{MATERIALS AND METHODS}

The restriction enzymes, purchased from New England Biolabs, Beverly, MA, were used in the buffer supplied by the manufacturer, unless specified. The enzymes, and all other reagents used in this study, were described previously (11). The 2'-Deoxyguanosine and 2'-deoxyadenosine were purchased from Sigma Chemical Co., St. Louis, MO. Most other reagents and solvents were purchased from Aldrich Chemical Co., Inc., Milwaukee, WI. The 7-bromomethylbenz[a]anthracene was prepared as described previously (12). The $N^{2}$-benzyl-2'-deoxyguanosine, $N^{6}$-benzyl-2'-deoxyadenosine, $N^{2}$-(benza[a]anthracen-7-ylmethyl)-2'-deoxyguanosine, $\quad N^{6}$-(benz[a]anthracen-7-ylmethyl)-2'-deoxyadenosine and the bulky aralkylated adducts, containing the 16-base oligodeoxyribonucleotides, were prepared as described previously (10).

\section{1) Synthesis and purification of oligonucleotides}

The 16-base oligodeoxyribonucleotides were prepared in an Applied Biosystems, Inc., Model 380B DNA synthesizer, and purified by polyacrylamide gel electrophoresis.

\section{2) Preparation of adduct containing plasmids}

Pairs of oligonucleotides were incorporated into the E. coli mutagenesis plasmid, pGP10 (Fig. 3), using the detailed procedure described previously $(11,13 \sim 15)$. In these constructions, the vector DNA was digested with $B s p$ MI restriction enzyme to remove the insert DNA sequence, as enclosed in the box, illustrated in Fig. 3. The major fragment produced during the $B s p$ MI digestion was isolated, and recircularized by ligating pairs of oligonucleotides, as illustrated in Fig. 3, into the plasmid where the insert had been. Following the recircularization, the covalently closed plasmids were isolated.

\section{3) Mutagenesis experiments in $E$. coli}

The E. coli strain, GP102 (15), is a lacZ alpha complementing version of the parent strain, AB1157 (16). Electroporation competent GP102 cells were prepared, and transformed with adduct containing, or control, plasmids, according to the procedure described previously (13). In experiments where SOS induction was required, $100 \mathrm{ml}$ of cells were grown to their mid log phase, as previously described (13), and then 
pelleted by centrifugation at $1,600 \mathrm{~g}$ for $5 \mathrm{~min}$ at room temperature. The resulting cell pellet was suspended in $25 \mathrm{ml}$ of "E" salts (17), transferred to a $100 \mathrm{~mm}$ sterile culture dish, with no lid, and irradiated with $75 \mathrm{~J} / \mathrm{m}^{2}$ of $254 \mathrm{~nm}$ light from a model UVGL-55 mineralight ${ }^{\circledR}$ lamp (UVP, Inc., San Gabriel, CA). This exposure had previously been determined to give a fifty percent survival of the GP102. The cells were re-pelleted under the same conditions, resuspended in $100 \mathrm{ml}$ of fresh LB broth and incubated, with shaking, at $37^{\circ} \mathrm{C}$ for $30 \mathrm{~min}$. The

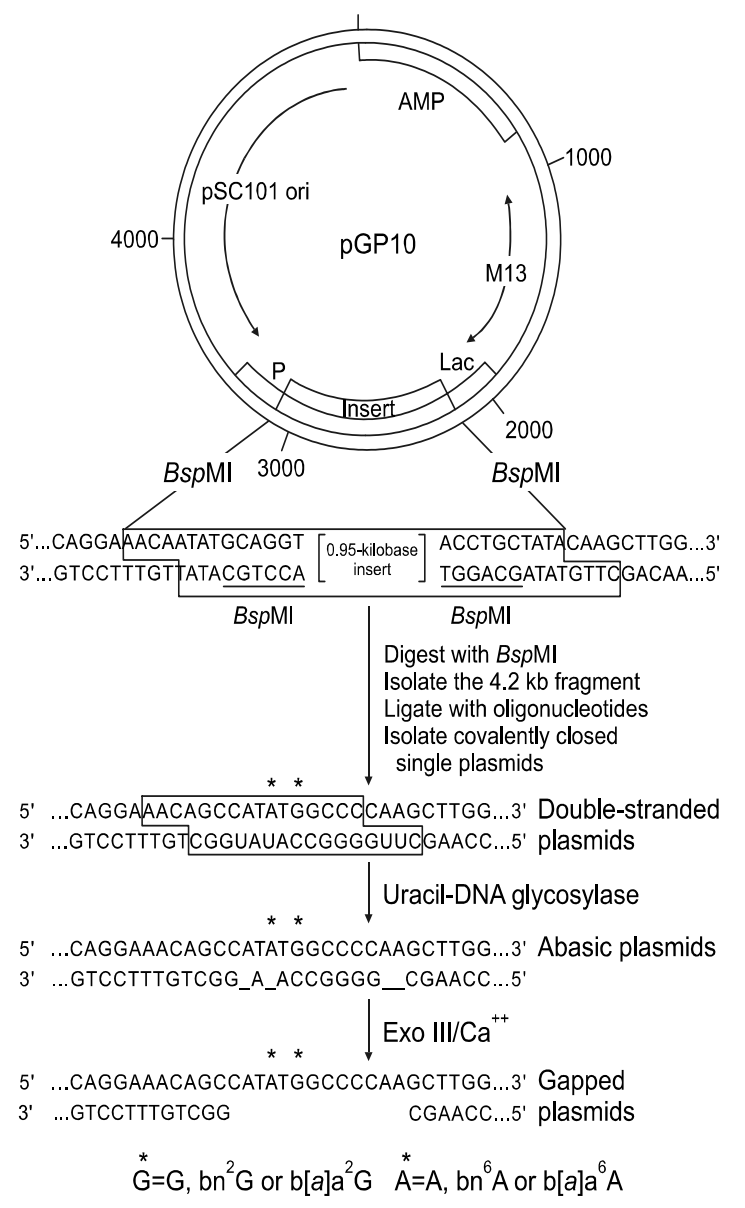

Fig. 3. pGP10 is a plasmid capable of replication in E. coli. The insert sequence was removed, by treatment with BspMI, and replaced by a pair of oligonucleotides. In this way, modified nucleotides were positioned within the ATG initiation codon of a lac ' ' gene. Adduct containing plasmids were used to transform a lac $Z^{\prime}$ a-complementing version of the $E$. coli strain, $\mathrm{AB} 1157$, and were plated onto media containing X-gal, IPTG and ampicillin. Adduct induced mutations give rise to white or sectored (blue and white) colonies, while non-mutant plasmids produce only blue colonies. The complementary strands of the adduct containing plasmids contained four deoxuyridines (as shown above) in place of the thymidines. Upon transformation into $E$. coli, the deoxyuridine containing plasmids were acted on by cellular uracil-DNA glycosylase and AP endonuclease, which created a gap in the complementary strand, across from the site of the nucleotide adduct. electroporation competent cells were then prepared, and transformed with the plasmids, as described previously (13). Following the electroporation and recovery, the bacteria were plated onto media containing X-gal and IPTG, and incubated as described in previously (15). The plates were then scored for blue and white colonies.

\section{4) Sequencing of mutant plasmids}

In order to identify the different kinds of lacZ inactivating mutations that occurred, the adduct containing and control plasmids, the white colonies, were isolated and subcultured. The plasmid DNA was isolated, and the region surrounding the lac $Z$ initiation codon sequenced using a dye terminator cycle sequencing kit (Perkin Elmer, Foster City, CA), with an Applied Biosystems 373A DNA sequencer (Foster City, CA).

\section{RESULTS}

\section{1) Mutagenicity in double-stranded plasmids}

A cassette plasmid permits monitoring of the mutagenicity of the carcinogen-modified bases in $E$. coli when they are positioned in the ATG initiation codon of the $\beta$-galactosidase gene (Fig. 3). This plasmid contains a nonfunctional lacZ' acomplementation gene, where the gene promotor $(\mathrm{P})$ is separated from the coding sequence (Lac) by a 0.95-kilobase insert. This insert is flanked by two recognition sequences for the restriction enzyme, BspMI, and treatment of the plasmid with this enzyme permits the removal of the insert sequence. The recircularization of the plasmid is accomplished by ligating the partially complementary 16-base sequence, created by the $B s p \mathrm{MI}$ digestion, to the stick ends of the major DNA fragment. The joining of the promoter, to the lacZ' coding sequence by a 16-base oligonucleotide containing the ATG initiation codon, results (Fig. 3). This system was used to measure the mutagenicities of the $b n^{2} G, b n^{6} A, b[a] a^{2} G$ and $b[a] a^{6} A$ residues that had been incorporated in place of the normal 2'-deoxyguanosine and 2'-deoxyadenosine of the ATG initiation codon.

The site-specific mutagenesis induced by these bulky adducts was monitored using the sectored colony assay system, as previously described (11). The adduct-induced mutations inactivate the gene, which led to the formation of blue and white colored colonies when the transformants from an a-comple-

Table 1. Relative transformation efficiency (RTE) from doublestranded plasmids in wild type $E$. coli

\begin{tabular}{lc}
\hline Adduct & RTE $(\%)^{\mathrm{a}}$ \\
\hline $\mathrm{G}$ or A & $100^{\mathrm{b}}$ \\
$\mathrm{bn}^{2} \mathrm{G}$ & $93.8 \pm 17.0$ \\
$\mathrm{bn}^{6} \mathrm{~A}$ & $107.2 \pm 24.3$ \\
$\mathrm{~b}[\mathrm{a}] \mathrm{a}^{2} \mathrm{G}$ & $84.6 \pm 10.8$ \\
$\mathrm{~b}[\mathrm{a}] \mathrm{a}^{6} \mathrm{~A}$ & $116.7 \pm 5.0$
\end{tabular}

${ }^{a}$ Mean \pm SD of four experiments. ${ }^{b}$ This value is defined as 100 for each experiment. The actual number of colonies ranged between 1500 and 4400. 


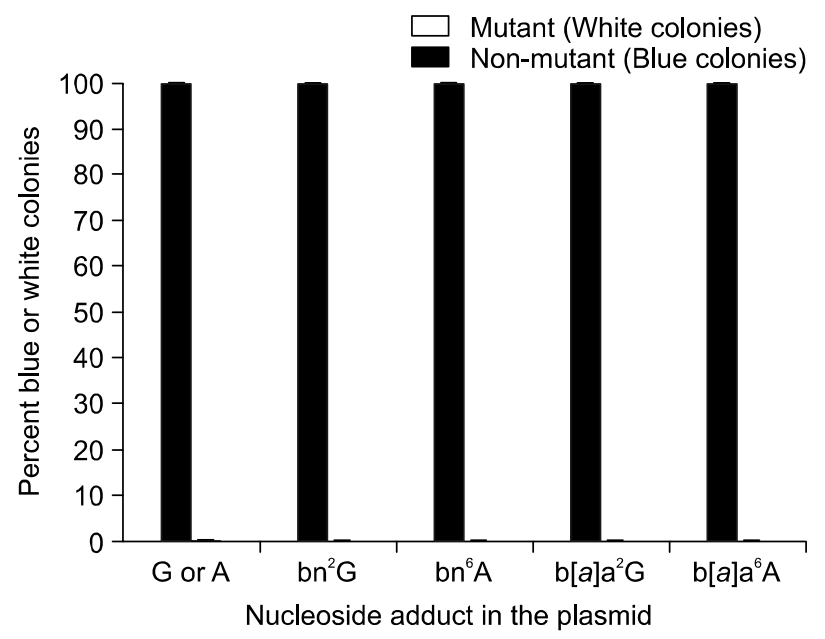

Fig. 4. Colony phenotypes resulting from aralkylated nucleosides in dU-containing plasmids. Each value represents the mean \pm SE of three determinations.

menting version of the $E$. coli strain, $\mathrm{AB} 1157$, are grown on media containing 5-bromo-4-chloro-3-indolyl $\beta$-D-galactoside (X-gal). In the absence of a mutation, blue colonies are produced (11). Table 1 shows the percentage of colonies produced by the double-stranded plasmids in wild type $E$. coli. There was no effect on the transformation efficiency, whether the double-stranded plasmids contained these bulky adducts, or not, indicating that none of these bulky adducts impedes the DNA replication. The percentages of mutant colonies produced by the modified plasmids were not significantly different from those produced by the unmodified plasmids (Fig. 4). The mutagenicities of the $b[a] a^{2} G$ and $b[a] a^{6} A$ were extremely low, which was totally unexpected. This study aimed to quantify the mutagenic potencies induced by these bulky adducts since 7-BrMeBA is a known mutagen and carcinogen. No mutagenicity for the $b n^{2} G$ and $b n^{6} A$ were detectable (Fig. 4).

\section{2) Mutagenicity in gapped plasmids}

The modified oligodeoxyribonucleotides were incorporated into double-stranded plasmids containing uracil residues, which led to the creation of a gap in the complementary strand due to the action of the E. coli uracil-DNA glycosylase. The E.coli containing the uracil-DNA glycosylase (UDG) and AP endonuclease would be expected to create a gap in the complementary strand, from the forcing of the replication past the adduct (13, 14) (Fig. 3). Failure to make a gap could result in a lack of mutagenicity. In order to make sure that a strand bias effect was not involved in the extremely low mutagenicity of the bulky adducts, a gap plasmid was intentionally created, in vitro, by treatment of a double-strand plasmid, with UDG and ExoIII in the presence of $\mathrm{Ca}^{++}$(Fig. 3). This externally created gap plasmid was transformed, and the mutagenicity re-evaluated for the same system. However, the mutagenicities of these adducts in the gapped plasmids were not increased at all compared to those of the double-strand plasmids (Fig. 5).

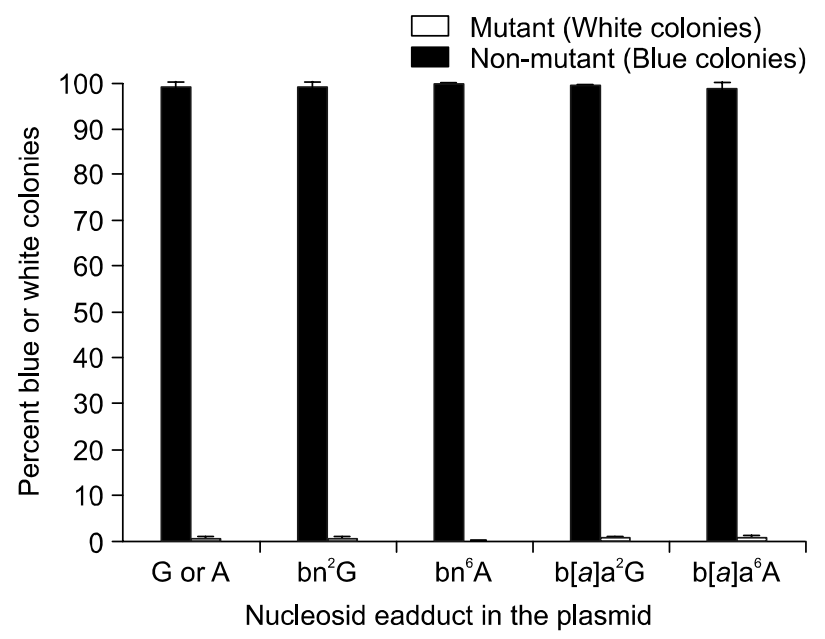

Fig. 5. Colony phenotypes resulting from aralkylated nucleosides in gapped plasmids, created in vitro, with, or without, SOS induction. Each value represents the mean \pm SE of three determinations.

\section{3) SOS induction}

SOS induction is commonly used for bulky adducts to observe increases in the mutagenicity (18). To test whether SOS induction had any effect on mutagenicities of the bulky adducts, it was induced by irradiating $E$. coli with UV light $(75 \mathrm{~J}, 254 \mathrm{~nm})$. However, the UV pretreatment of these adducts in $E$. coli showed no distinguishable change in the mutagenicities compared to that of the control (Fig. 5). The SOS induction was considered to have no measurable effects on the mutagenicities of the adducts.

\section{4) Mutations in E. coli}

Following the transformation, with the control and adduct containing plasmids, ten white colonies were chosen. The plasmid DNA was isolated and sequenced. The majority of the identified mutations were most likely artifacts of the plasmid manipulation. In the case of $b[a] a^{2} G$, one G-to-C and one $\mathrm{G}$-to-T transversions, and in the case of $\mathrm{b}[\mathrm{a}] \mathrm{a}^{6} \mathrm{~A}$, a single A-to- $\mathrm{T}$ transversion, were observed at the site of the adduct. No base changes in, or near, the initiation codon could be reliably attributed to the presence of either the $b^{2} \mathrm{G}$ or $b n^{6} \mathrm{~A}$.

\section{DISCUSSION}

The primary objective of this study was to examine the site-specific mutagenicity in E. coli of the major DNA adducts produced by the aralkylating agent, 7-(bromomethyl)benz[a]anthracene, i.e., $b[a] a^{2} \mathrm{G}$ and $\mathrm{b}[\mathrm{a}] \mathrm{a}^{6} \mathrm{~A}$, when the modified base was incorporated into either a double-stranded or gapped plasmid vector. We then compare these data to those for the analogous, but simpler benzylated derivatives of guanine and adenine, to observe the effect of increasing the size of the exocyclic amino group substituent on the mutagenic potency, and how a substituted structure can influence the mutagenicity of bulky exocyclic amino-substituted adducts. Unfortunately, 
however, the four aralkylated bulky adducts revealed no mutagenic potencies (Fig. 4 and 5), even though 7-(bromomethyl)benz[a]anthracene is a known carcinogen $(4 \sim 7)$.

It has been assumed that the notable low frequency of mutations induced by adducts, in site-specific mutagenesis studies, resulted from the efficient repair of the adduct. The lack in a mutation frequency observed for the $b n^{2} \mathrm{G}, b n^{6} \mathrm{~A}, \mathrm{~b}[\mathrm{a}] \mathrm{a}^{2} \mathrm{G}$ and $\mathrm{b}[\mathrm{a}] \mathrm{a}^{6} \mathrm{~A}$ residues might be as a result of repairs. However, this seems an unlikely explanation since active excision repairs would not be expected on gapped plasmids containing four aralkylated bulky adducts $(13,15)$.

Several studies have shown marked differences in the mutagenic potencies of the exocyclic DNA adducts [e.g., $1, N^{2}$ ethenodeoxyguanosine, $N^{2}$,3-ethenodeoxyguanosine, $3, N^{4}$-ethenodeoxycytidine and $1, N^{6}$-ethenodeoxyadenosine] produced by chloroethylene oxide and chloroacetaldehyde, which are reactive metabolites of the human carcinogen, vinyl chloride, between $E$. coli and mammalian cells $(19 \sim 21)$. 1, $N^{6}$-Ethenodeoxyadenosine, one of the four exocyclic DNA adducts, was highly mutagenic in mammalian cells, but only marginally mutagenic in E. coli (19). 1, $N^{2}$-ethenodeoxyguanosine was highly mutagenic in $E$. coli, but only weakly mutagenic in mammalian cells. For $3, N^{4}$-ethenodeoxycytidine, a converse relationship was observed (20). These data strongly suggest that the sitespecific mutagenesis of $b n^{2} G, b n^{6} A, b[a] a^{2} G$ and $b[a] a^{6} A$ should be examined in human cells.

The sequence context has profoundly influenced the mutagenic potencies of some bulky adducts in site-specific mutagenesis studies $(22 \sim 25)$. This sequence context effect may be the reason why the bulky aralkylated adducts showed no significant mutagenicities in E. coli.

\section{CONCLUSIONS}

In this $E$. coli site-specific mutagenesis system, none of the aralkylated adducts exhibited any significant genotoxicity or mutagenicity. A SOS induction had no measurable effect on the mutagenicity of these adducts. The low genotoxicity and mutagenicity of these adducts may be either intrinsic to their structure, or as a result of a sequence context effect, in this system. Further studies are in progress to elucidate the mechanism by which these aralkylated adducts exert no mutagenic effect in E. coli. Other studies will also be required to determine the mutagenicity of these same adducts in human cells.

\section{REFERENCES}

1. Basu AK, Essigmann JM. Site-specifically modified oligodeoxynucleotides as probes for the structural and biological effects of DNA-damaging agents. Chem Res Toxicol 1988;1: 1-18.

2. Pauly GT, Powers M, Pei GK, Moschel RC. Synthesis and properties of H-ras DNA sequences containing $\mathrm{O}^{6}$-substituted 2'-deoxyguanosine residues at the first, second, or both positions of codon 12. Chem Res Toxicol 1988;1:391-397.

3. Moschel RC. Reaction of aralkyl halides with nucleic acid components and DNA. In: Hemminki K, Dipple A, Shuker DEG, Kadlubar FF, Segerback, Bartsch H, eds. DNA Adducts
Identification and Biological Significance. IARC Scientific Publication No. 128. France: IARC, Lyon, 1994:25-36.

4. Dipple A, Brookes P, Mackintosh DS, Rayman MP. Reaction of 7-bromomethylbenz[a]anthracene with nucleic acids, polynucleotides, and nucleosides. Biochemistry 1971;10:4323-4330.

5. Kim KK. Expression of cyclinD1 and CDK4 in DMBA-induced rat ovarian cancer. Cancer Res Treat 2001;33:229-235.

6. Dipple A, Slade TA. Structure and activity in chemical carcinogenesis: Studies of variously substituted 7-bromomethylbenz[a]anthracenes. Eur J Cancer 1971;7:473-476.

7. Rayman MP, Dipple A. Structure and activity in chemical carcinogenesis. Comparison of the reactions of 7-bromomethylbenz[a]anthracene and 7-bromomethyl-12-methylbenz[a]anthracene with deoxyribonucleic acid in vitro. Biochemistry 1973;12:1202-1207.

8. Moon KY, Moschel RC. Effect of ionic state of 2'-deoxyguanosine and solvent on its aralkylation by benzyl bromide. Chem Res Toxicol 1998;11:696-702.

9. Moon KY, Kim YS. A one-pot preparation of 1-benzyl-2'deoxyinosine from ionized 2'-deoxyinosine. Nucleosides $\mathrm{Nu}-$ cleotides Nucleic Acids 2000;19(7):1135-1143.

10. Moon KY, Kim YS. Synthesis and characterization of oligonucleotides containing site-specific bulky $\mathrm{N}^{2}$-aralkylated guanines and $\mathrm{N}^{6}$-aralkylated adenines. Arch Pharm Res 2000; 23:139-146.

11. Pauly GT, Hughes SH, Moschel RC. A Sectored colony assay for monitoring mutagenesis by specific carcinogen-DNA adducts in Escherichia coli. Biochemistry 1991;30:11700-11706.

12. Pei GK, Moschel RC. Aralkylation of 2'-deoxyguanosine: Medium effects on sites of reaction with 7-(bromomethyl)benz[a]anthracene. Chem Res Toxicol 1990;3:292-295.

13. Pauly GT, Hughes SH, Moschel RC. Mutagenesis in Escherichia coli by three $\mathrm{O}^{6}$-substituted guanines in double-stranded or gapped plasmids. Biochemistry 1995;34:8924-8930.

14. Pauly GT, Hughes SH, Moschel RC. Comparison of mutagenesis by $O^{6}$-methyl-and $O^{6}$-ethylguanine and $O^{4}$-methylthymine in Escherichia coli using double-stranded and gapped plasmids. Carcinogenesis 1998;19:457-461.

15. Pauly GT, Hughes SH, Moschel RC. Response of repaircompetent and repair-deficient Escherichia coli to three $O^{6}$ substituted guanines and involvement of methyl-directed mismatch repair in the processing of $O^{6}$-methylguanine residues. Biochemistry 1994;33:9169-9177.

16. DeWitt SK, Adelberg EA. The occurrence of a genetic transposition in a strain of escherichia coli. Genetics 1962;47: 577-585.

17. Vogel HJ, Bonner DM. Acetylornithinase of escherichia coli: Partial purification and some properties. J Biol Chem 1956; 218:97-106.

18. Walker GC. Mutagenesis and inducible responses to deoxyribonucleic acid damage in Escherichia coli. Microbiological Rev 1984;48:60-93.

19. Pandya GA, Moriya M. $1, N^{6}$-Ethenodeoxyadenosine, a DNA adducts highly mutagenic in mammalian cells. Biochemistry 1996;35:11487-11492.

20. Moriya M, Zhang W, Johnson F, Grollman AP. Mutagenic potency of exocyclic DNA adducts: Marked differences between Escherichia coli and simian kidney cells. Proc Natl Acad Sci 1994;91:11899-11903.

21. Moriya M, Spiegel S, Fernandes A, Amin S, Liu T, Geacintov N, Grollman AP. Fidelity of translesional synthesis past benzo [a]pyrene diol epoxide-2'-deoxyguanosine DNA adducts: Marked effects of host cell, sequence context, and chirality. Biochemistry 1996;35:16646-16651.

22. Rodriguez H, Loechler EL. Mutational specificity of the (+)anti-diol epoxide of benzo[a]pyrene in a sup $F$ gene of an 
80 Cancer Research and Treatment 2003;35(1)

Escherichia coli plasmid: DNA sequence context influences hotspots, mutagenic specificity and the extent of SOS enhancement of mutagenesis. Carcinogenesis 1993;14:373-383.

23. Litinski V, Chenna A, Sagi J, Singer B. Sequence context is an important determinant in the mutagenic potential of 1 , $\mathrm{N}^{6}$-ethenodeoxyadenosine $(\varepsilon \mathrm{A})$ : formation of $\varepsilon \mathrm{A}$ basepairs and elongation in defined templates. Carcinogenesis 1997;18:16091615 .
24. Khalili H, Zhang FJ, Harvey RG, Dipple A. Mutagenicity of benzo[a]pyrene-deoxyadenosine adducts in a sequence context derived from the p53 gene. Mutat Res 2000;465:39-44.

25. Ponten I, Sayer JM, Pilcher AS, Yagi H, Kumar S, Jerina DM, Dipple A. Sequence context effects on mutational properties of cis-opened benzo[c]phenanthrene diol epoxide-deoxyadenosine adducts in site-specific mutation studies. Biochemistry 1999;38:1144-1152. 